\title{
Emerging infectious diseases and animal social systems
}

\author{
Charles L. Nunn · Peter H. Thrall · Kelly Stewart · Alexander H. Harcourt
}

Received: 21 November 2005/ Accepted: 16 April 2007/Published online: 30 May 2007

(C) Springer Science+Business Media B.V. 2007

\begin{abstract}
Emerging infectious diseases threaten a wide diversity of animals, and important questions remain concerning disease emergence in socially structured populations. We developed a spatially explicit simulation model to investigate whether-and under what conditions-disease-related mortality can impact rates of pathogen spread in populations of polygynous groups. Specifically, we investigated whether pathogen-mediated dispersal (PMD) can occur when females disperse after the resident male dies from disease, thus carrying infections to new groups. We also examined the effects of incubation period and virulence, host mortality and rates of background dispersal, and we used the model to investigate the spread of the virus responsible for Ebola hemorrhagic fever, which currently is devastating African ape populations. Output was analyzed using regression trees, which enable exploration of hierarchical and non-linear relationships. Analyses revealed that the incidence of disease in single-male (polygynous) groups was significantly greater for those groups containing an average of more than six females, while the total number of infected hosts in the population was most sensitive to the number of females per group. Thus, as expected, PMD occurs in polygynous groups and its effects increase as harem size (the number of females) increases. Simulation output further indicated that population-level effects of Ebola are likely to differ among multi-male-multi-female chimpanzees and polygynous gorillas, with larger overall numbers of chimpanzees infected, but more gorilla groups becoming infected due to increased dispersal when the
\end{abstract}

C. L. Nunn (ه)

Max Planck Institute for Evolutionary Anthropology, 04103 Leipzig, Germany

e-mail: nunn@eva.mpg.de

C. L. Nunn

Department of Integrative Biology, University of California, Berkeley, CA 94720-3140, USA

P. H. Thrall

CSIRO Plant Industry, GPO Box 1600, Canberra, ACT 2601, Australia

K. Stewart - A. H. Harcourt

Department of Anthropology, University of California, Davis, CA 95616, USA 
resident male dies. Collectively, our results highlight the importance of social system on the spread of disease in wild mammals.

Keywords Social systems - Emerging infectious disease - Individual-based model · Ecological simulation · African apes · Ebola hemorrhagic fever · Conservation

\section{Introduction}

A number of infectious diseases are threats to populations of threatened animal species (Daszak et al. 2000; Dobson and Foufopoulos 2001; Cleaveland et al. 2002; Nunn and Altizer 2006). For example, canine distemper virus has had devastating effects on African wild dogs and lions, as well as on aquatic mammals such as seals and porpoises (Osterhaus et al. 1995; Roelke-Parker et al. 1996; Kennedy et al. 2000; Funk et al. 2001), and outbreaks of Ebola hemorrhagic fever have drastically impacted populations of African apes (Walsh et al. 2003; Leroy et al. 2004; Pourrut et al. 2005; Bermejo et al. 2006). Similarly, periodic eruptions of rinderpest have had adverse effects on a wide range of ungulates (Rossiter 2001), with buffalo mortality approaching $80 \%$ in some populations during a recent epidemic of rinderpest (Kock et al. 1999), and the ongoing decline of amphibian populations has been linked with disease outbreaks caused by a chytridiomycete fungus (Berger et al. 1998; Daszak et al. 1999, 2003).

Studies of disease emergence have focused mainly on sources of infection, such as spillover from reservoir hosts to vulnerable hosts of different species (Daszak et al. 2000; Woolhouse et al. 2005). In the case of canine distemper virus, spillover is thought to occur from overlapping populations of domesticated dogs (Alexander and Appel 1994; RoelkeParker et al. 1996; Carpenter et al. 1998), whereas livestock are likely to serve as the source of infections involving rinderpest (Rossiter 2001). Once pathogen spillover occurs, however, contact among conspecifics will influence the degree to which a socially transmitted pathogen becomes established in a population (Anderson and May 1979, Morse 1995; Woolhouse et al. 2005).

Despite the recognition that social and mating behavior are major determinants of contact patterns (and therefore disease transmission opportunities) within host populations, the links between disease emergence and host characteristics, including group composition, sexual contact patterns, and population density, have had an under-appreciated role in studies of disease emergence (Thrall et al. 1998; Altizer et al. 2003; Nunn and Altizer 2006). Characteristics of infection in individual hosts, such as incubation period or transmission mode, will further impact the ability of a novel infectious agent to become established in a host population. In a socially structured population, for example, a longer incubation period increases the chances that an individual will immigrate into another group before pathology occurs, enabling the pathogen to overcome limits on disease spread when populations are subdivided into stable social groups (Loehle 1995; Cross et al. 2005).

We developed an individual-based simulation model to investigate host and pathogen traits that are likely to influence the establishment and spread of a virulent and directly transmitted pathogen, such as rabies or Ebola, introduced to a susceptible host population. We apply this model to primates, but the model should also apply more broadly to any socially structured host population in which social groups are relatively stable, with male or female dispersal between groups. 
We were particularly interested in using the model to test the "pathogen-mediated dispersal" hypothesis (PMD). Pathogen-mediated dispersal is based on the idea that when the single male of a polygynous group dies, females could disperse and carry the disease with them to new groups, thus increasing the rate of spread at the population level. While males are also likely to die in a polygynandrous group, complete extinction of males is less likely, and movement of infected females from the group is therefore also less likely. Pathogen-mediated dispersal predicts that an introduced infectious disease will reach more social groups when those groups are polygynous, and when there are more females per male in polygnous groups (since this will create more dispersing individuals if the male dies from disease). Understanding the conditions under which PMD increases rates of infection and mortality could be essential to controlling the spread of infectious disease, especially in socially structured populations where dispersal is a major determinant of disease spread (Loehle 1995; Thrall et al. 1998; Cross et al. 2005; Nunn and Altizer 2006). Moreover, PMD highlights that under some circumstances, group composition - the number of males and females counted separately-can be more important for understanding disease risk than overall group size-i.e., the total number of males and females.

In our simulation model, dispersal between groups occurred locally by individuals based on a per-day probability of movement to new groups, or when all members of the opposite sex disappeared from a group, for example through disease-induced mortality. We were particularly interested in examining the spread of infection in a spatial context, given that individuals should have more difficulty finding groups during dispersal as the density of groups declines, thereby potentially slowing the spread of disease. Indeed, a number of previous studies have shown that spatial structure can significantly impact disease dynamics and patterns of virulence, as well as longer-term evolutionary processes (Thrall and Antonovics 1995; Gandon et al. 1996; Boots and Sasaki 1999; Roy and Kirchner 2000; Carlsson-Granér and Thrall 2002; O'Keefe and Antonovics 2002).

We also investigated the effect of several epidemiological parameters, including incubation period, disease-induced mortality, rates of dispersal, and the probability of transmission among individuals within groups, as these will interact with host behavioral patterns to determine whether a pathogen can persist when a population is composed of stable social groups (Cross et al. 2005). We note that our focus is on pathogens that are transmitted through direct social contacts rather than sexually transmitted diseases, which may be favored under different conditions (Lockhart et al. 1996; Thrall et al. 1998). We further note that virulence refers to the negative effects of infection on host fitness, with increasing virulence correlated with increased probability of host death.

In addition to investigating the general conditions that increase the spread of a novel virulent pathogen, we applied our approach to investigate a real-world case involving the spread of Ebola hemorrhagic fever in gorillas (Gorilla gorilla) and chimpanzees (Pan troglodytes, Formenty et al. 1999a; Walsh et al. 2003; Leroy et al. 2004; Bermejo et al. 2006). This host-pathogen system is of particular interest in the context of our model because chimpanzees live in multi-male, multi-female communities (a polygynandrous mating system), whereas gorillas live in smaller groups in which a single male (or sometimes two males) defend access to a group of females (a largely polygynous mating system). Both species are well studied, which provides the detailed information needed to parameterize the simulation model. 


\section{Methods}

\section{Simulation model structure}

We developed a model using the computer package MATLAB (version 7, Natwick, MA) to simulate the epidemic spread of infectious disease in a structured host population composed of multiple mating groups. For each run of the simulation, groups of susceptible individuals were formed based on user-specified values for the expected number of males and females in groups. This allowed us to simulate mating systems ranging from polygyny to multi-male multi-female group structures. A key assumption of the model is that transmission occurs entirely within groups, rather than during encounters between groups, through contact with infectious material in areas of range overlap, or via other mechanisms of group-to-group contact (Walsh et al. 2007). While these other forms of inter-group spread will influence prevalence, and are therefore important for making predictions about disease spread in different populations and species, our expectation is that these mechanisms will have no qualitative effects on tests of the PMD hypothesis.

Infection was initiated in a single female, and the spread of disease was tracked through the population in one-day time steps. In all simulations, this daily cycle was shorter than the duration of infection in an individual host. In each time step, individuals remained in their original reproductive group or dispersed to other groups in the population, as determined by a sex-specific probability of dispersal. During the dispersal phase, individuals were placed in a floater pool (i.e., solitary individuals lacking contact with other hosts). Individuals that died as a result of infection were not replaced, so that population size typically declined as the infection spread through the population. This situation is representative of emerging diseases as well as those involved in periodic outbreaks (e.g., rinderpest in African ruminants), where dramatic reductions in host numbers are often the primary short-term consequence of an epidemic. By using a simulation approach, we were able to evaluate the role of stochastic factors during population decline (we note that complete extinction of host populations did not occur in any of the simulations).

Susceptible individuals that became infected passed through the following stages: infected/non-infectious (incubation period), infected/infectious (infectious period), and recovered or dead (Fig. 1). Recovered individuals were assumed to be permanently im-

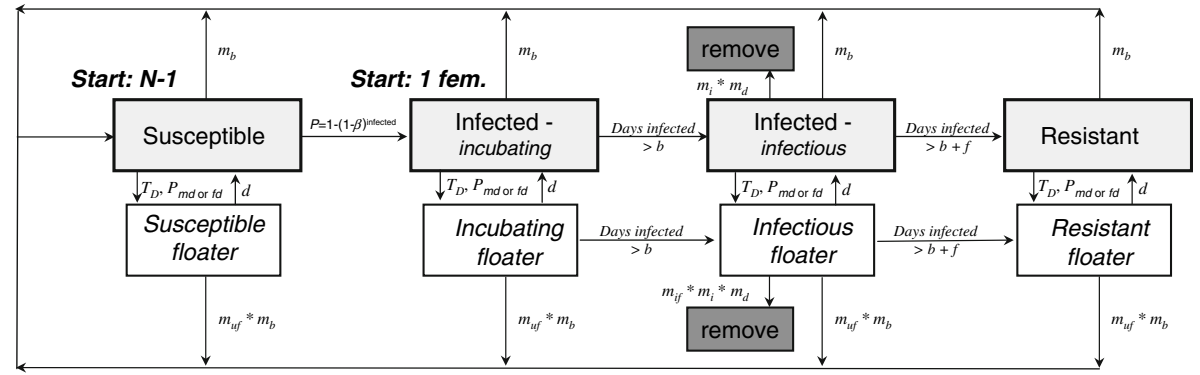

Fig. 1 Schematic diagram of the simulation model. Variables correspond to Table 1. After initialization of social groups, the simulation starts with $N-1$ individuals susceptible to infection and a single infected female. Individuals that become infected pass through three stages, ending in resistance or death and removal from the population. Gray boxes show individuals in groups, while open boxes represent floaters that are unassociated with a reproductive group. Susceptible floaters cannot become infected, but infected floaters pass through the stages of infection in the same way as individuals in groups. Background mortality results in the birth of a new individual of the same sex in a randomly selected group 
mune to re-infection. Because susceptible floaters lacked contact with conspecifics, there was no potential for them to become infected until they successfully entered a reproductive group; individuals infected prior to entering the floater pool passed through the noninfectious, infectious and recovered stages, potentially carrying the pathogen to a new social group (Fig. 1). Output for each run included statistics on the number of individuals and social groups that were infected, disease-induced mortality, and number of times that infected individuals dispersed. A simulation run was initiated with a set of parameter values drawn randomly from pre-determined ranges (random sampling, see below). Simulation runs were terminated when the prevalence of infection in a host population declined to zero (this occurred in all cases simulated here).

\section{Group formation}

The simulation model takes as input total population size $(N)$ and the mean number of females $\left(g_{\mathrm{f}}\right)$ and males $\left(g_{\mathrm{m}}\right)$ in social groups (Table 1). The starting total population size was set to $N=2,000$ individuals in all simulations (except when applied to Ebola in apes, see below). Simulations were initiated by repeatedly forming groups of males and females until the total population size was reached [i.e., within $N \pm\left(g_{\mathrm{m}}+g_{\mathrm{f}}\right) / 2$ ]. These groups were then placed randomly on the smallest square grid that could support the number of groups produced. Thus, the size of the matrix was a function of the number of groups, and the grid

Table 1 Definitions of model parameters and symbols, together with ranges of parameter values investigated in the simulation

\begin{tabular}{lll}
\hline Parameter & Definition & Numerical range $^{\mathrm{a}}$ \\
\hline$b$ & $\begin{array}{l}\text { Incubation period (individuals are infected but non-infectious, integer } \\
\text { values) }\end{array}$ & $1-10$ days \\
$f$ & Infectious period & 9 days \\
$N$ & Total population size & 2,000 \\
$g_{\mathrm{f}}$ & Average number of females in groups (integer values) & $1-10$ \\
$g_{\mathrm{m}}$ & Average number of males in groups (integer values) & $1-5$ ( $\left._{\mathrm{m}} \leq \mathrm{g}_{\mathrm{f}}\right)$ \\
$d$ & Minimum days in the floater pool when either $P_{\mathrm{md}}$ or $P_{\mathrm{fd}}>0$ & 2 \\
$P_{\mathrm{fd}}$ & Baseline probability of female dispersal per day & $0 \leq \mathrm{P}_{\mathrm{fd}} \leq 0.005$ \\
$P_{\mathrm{md}}$ & Baseline probability of male dispersal per day & $0 \leq \mathrm{P}_{\mathrm{md}} \leq 0.005$ \\
$T_{\mathrm{D}}$ & Critical threshold for dispersal (same for both sexes) & 0 \\
$\beta$ & Per-contact transmission probability & $0-0.8$ \\
$m_{\mathrm{b}}$ & Baseline disease-independent mortality rate per day & $0-0.001$ \\
$m_{\mathrm{i}}$ & Mortality multiplier for an infectious individual in a group \\
$m_{\mathrm{d}}$ & Disease-induced mortality, normally distributed across the infectious period & $0-0.5$ \\
$m_{\mathrm{if}}$ & $\quad f$ with peak at midpoint and variance of $f / 4$ & \\
$m_{\mathrm{uf}}$ & Mortality multiplier for an infected-infectious floater, multiplied by $m_{\mathrm{i}}$ & 4 \\
& Mortality multiplier for an uninfected, immune or incubating floater, & 3 \\
$\mathrm{a}$ & multiplied by $m_{\mathrm{b}}$ &
\end{tabular}

\footnotetext{
${ }^{a}$ When a range is given, values were selected randomly (random sampling) within the range for the main set of simulations

b This value is multiplied by probability of death, $m_{\mathrm{d}}$, which varies over the course of infection following a normal distribution. When this multiplier $=1$, the peak probability of death at the midpoint of the infectious period is 0.5
} 
was not always filled. Across a subset of 30,000 runs used in Analysis 1 described below, simulated populations were represented by a median of 225 cells (a $15 \times 15$ matrix), ranging from 121 to 1,024 cells, with a median of 14 empty cells at the beginning of simulations (range 0-62). Thus, approximately $6 \%$ of the cells were empty in a typical simulation.

Within each group, the numbers of each sex were obtained as random draws from a Poisson distribution. For males, this distribution was centered on the user-defined mean number of males per group $\left(g_{\mathrm{m}}\right.$, Table 1$)$. The minimum value was 1 and the distribution had a variance of $g_{\mathrm{m}}-1+0.02^{\mathrm{gm}}$. This adjustment was implemented to produce a low (but non-zero) variance at the smallest group sizes, because even in so-called monogamous species, such as gibbons, some groups have more than one male (Reichard 2003). Such situations could greatly impact the potential for PMD to occur, and were therefore important to consider in the model.

After obtaining a value from the Poisson distribution for the number of males in a group, we used this value and the expected sex ratio (ratio of user-defined parameters $g_{\mathrm{f}}$ to $\left.g_{\mathrm{m}}\right)$ to identify the expected number of females in that group. We then used the Poisson distribution to calculate the actual number of females in a particular group, with variance equal to $g_{\mathrm{f}}-1+0.02^{g \mathrm{f}}$. This approach therefore established a correlation between the number of males and females in groups, as is commonly observed in social mammals (e.g., primates, Andelman 1986; Altmann 1990, 1999; Mitani et al. 1996; Nunn 1999; Lindenfors et al. 2004).

Disease spread and the course of infection in individuals

We investigated several parameters that are typically considered to be pathogen characteristics, although in reality they reflect genetic and ecological aspects of the interaction between host and pathogen. The transmission parameter $(\beta)$ reflects the overall probability of a given infected host spreading the disease to another susceptible individual within the same group. Inter-individual contact rates were assumed to be unaffected by group size and equal among all individuals within a social group (i.e., transmission rates between and within sexes were the same; age classes were not included in this model). Thus, overall contact rate increased with group size, as predicted by standard mass-action epidemiological models (May and Anderson 1979; Anderson and May 1981). Using the binomial distribution, the probability of a susceptible individual not becoming infected as a result of contacts with members of its social group is given by $(1-\beta)^{I}$, where $I$ represents the number of infectious individuals in that group. Thus, the overall probability of infection for an individual is given by $1-(1-\beta)^{I}$.

Following initial infection, individuals entered an incubation period of duration $b$. During incubation, hosts were not infectious, and there was no added mortality due to infection (i.e., a latent period). We ran the general simulations with $b$ ranging between 1 and 10 days (Table 1), which fits expectations for some real-world emerging diseases. For canine distemper, for example, the shedding of virus begins approximately 7 days post infection (Appel and Summers 1995), while Ebola has an incubation period of approximately 8 days and individuals may not be fully recovered for up to 6 weeks after symptoms are initially expressed (Formenty et al. 1999b; Pourrut et al. 2005). After incubation, the pathogen entered an infectious period of $f$ days during which the pathogen could be transmitted to another host individual and infected hosts experienced disease-related mortality (as described in the next section). We ran simulations with $f$ held constant at 
9 days (Table 1), under the assumption that highly pathogenic diseases should be more sensitive to variation in incubation period, i.e., before disease symptoms and increased mortality are expressed.

\section{Host mortality}

Individual hosts were subject to two sources of mortality, background mortality $\left(m_{\mathrm{b}}\right)$ and disease-related mortality $\left(m_{\mathrm{d}}\right.$, based on the time-course of infection and a multiplier, $m_{\mathrm{i}}$, described below). We assumed that deaths from background sources of mortality, such as predation and old age, were balanced by births. Thus, for background mortality, a healthy individual of the same sex replaced an individual that died, and in the absence of diseaserelated mortality, population growth would be zero. Newly generated healthy individuals were randomly assigned to existing reproductive groups. This procedure, along with dispersal procedures described below, will tend to erase the initial correlation between male and female numbers, but should have little effect on the short time scales of simulations conducted here (see Results). Although background death rates were low (Table 1) relative to disease-related mortality, we felt that it was important to include background mortality to ascertain if such mortality has an effect on the dynamics of disease under conditions typical of highly pathogenic diseases in long-lived hosts, such as primates.

Over the infectious period, the per-day probability of death was represented as a normal distribution with the maximum probability of death set at 0.5 and centered at the midpoint of the infectious period (i.e., day 5 when $f=9$ ), with variance equal to $1 / 4$ of the total duration of infectiousness (i.e., f/4). Because many infections have a characteristic build up and recovery period prior to and after the period of maximum mortality, this approach is likely to be more realistic than simply assuming a constant elevated probability of death during the infectious period. We scaled this probability by multiplying it by $m_{\mathrm{i}}$, which took random values from 0 to 1 (Table 1 ). With the nine-day infectious period used here, the actual probability of death due to disease was maximized at $72.7 \%$ (with $m_{\mathrm{i}}=1$ ), with the highest per-day probability on day 5; for the midpoint of the range of values used for $m_{\mathrm{i}}$ $(=0.5)$, the probability of death over the infectious period was $43.8 \%$. Thus, most simulations involved a highly virulent pathogen with relatively low chances for recovery.

\section{Host dispersal}

In many mammals, one or both sexes can disperse, and dispersal rates often differ between the sexes. We investigated the effect of sex-biased dispersal $\left(P_{\mathrm{fd}}\right.$ and $P_{\mathrm{md}}$ for females and males, respectively), measured as the per-day probability that an individual disperses from a group (Table 1). Dispersing individuals moved in a random walk until contact was made with a new group. Each day, individuals could move to any of the surrounding cells, including those on the diagonal, or they could stay at their current locations, with equal probabilities for these nine possibilities. The lattice was bounded, as is typical in a reserve or park surrounded by human populations.

When floaters found a group with one or more members of the opposite sex, they became linked to that group, but not actually members of the group until at least $d$ days had passed since they had entered the floating pool. This assumption is realistic to the extent that integration into a group is likely to be fast but is unlikely to be instantaneous, and because dispersal times should increase when local density declines and groups are more 
difficult to locate. Thus, if a dispersing individual did not encounter a group, the actual number of days in the floater pool could exceed the user-specified value of $d$. Further note that when more than one individual dispersed from a particular group they did so independently. Thus, while multiple individuals from the same group were more likely to join the same group than floaters from different groups, this was decided on an individual basis through independent random walks by each floater. Individuals could not enter the group from which they most recently originated.

In addition to background dispersal, individuals dispersed when numbers of the opposite sex fell below a critical threshold $\left(T_{\mathrm{D}}\right)$. For all simulations, we set this threshold to a conservative and plausible level: individuals always dispersed when they lacked mating partners, i.e., when numbers of the other sex fell to zero $\left(T_{\mathrm{D}}=0\right)$. Females are known to disperse to new groups when the number of males declines; in one primate study, for example, all males in a chacma baboon (Papio ursinus) group died from an unknown pathogen, and the females of that group fused with a neighboring group (Barrett and Henzi 1998). Similarly, gorilla females can disperse rapidly to new groups when the silverback of their original group dies (Watts 1989; Stokes et al. 2003).

Although infection status had no impact on the probability of individual dispersal or the duration of dispersal, we assumed that individuals that were infectious experienced a higher probability of dying while dispersing, as might be expected given that such individuals would be in less familiar habitat in which it could be difficult to locate resources or refuges for recovery. Data on the behavior and mortality of dispersing animals are notoriously difficult to obtain (Slatkin and Hausfater 1976). Thus, we set the increased probability of death during infection to a value of $m_{\mathrm{if}}=4$, which was multiplied by $m_{\mathrm{i}}$ and the value from the normal distribution described above $\left(m_{\mathrm{d}}\right)$ to obtain a probability of death for infectious individuals during dispersal. Uninfected, immune and incubating hosts also experienced a higher rate of mortality during dispersal $\left(m_{\mathrm{uf}}\right)$, set in all runs at three times the baseline probability of death $m_{\mathrm{b}}$.

Analysis of simulation output

Output from the simulation was analyzed using regression trees (De'ath and Fabricius 2000; Roff and Roff 2003), and in some cases with more standard statistical approaches, such as ANOVA. Regression trees were calculated using the Statistics Toolbox in MATLAB v. 7.0. The goal of using this approach was to describe how variables interact to influence several key aspects of disease spread, as described below, and to estimate how much variation a set of parameters could explain in our stochastic simulation model. We split impure nodes when the number of observations for that node was 500 (for analyses of scenarios based on 30,000 simulation runs) or 1,000 (for 60,000 runs). After creating an initial tree using the simulation output, we used 10-fold cross-validation to identify the pruning level with the minimal cost (De'ath and Fabricius 2000), identified as the tree with the minimum error rate. Using this pruned tree, we calculated the percentage of variance explained by comparing predicted and observed values.

We conducted two sets of regression tree analyses to investigate the effect of a suite of predictor variables on four output variables involving number of infections in the population, disease-related deaths, number of groups infected, and number of times that infected individuals dispersed from groups. In Analysis 1, we investigated patterns of infection in simulations in which either sex dispersed in the spatial model $(n=30,000$ runs). We investigated seven predictor variables: number of females per group $\left(g_{\mathrm{f}}\right)$, 
one-male versus many-males per group (categorical variable reflecting mating system), summed dispersal rate of males and females $\left(P_{\mathrm{md}}+P_{\mathrm{fd}}\right)$, baseline mortality $\left(m_{\mathrm{b}}\right)$, disease-related mortality $\left(m_{\mathrm{i}}\right)$, incubation period $(b)$, and per-contact transmission probability $(\beta)$.

In Analysis 2, we investigated whether male-only or female-only migration had an impact on the same four dependent variables. We combined data from spatial simulations with only male or female dispersal ( $n=60,000$ runs total) and investigated eight predictor variables: the number of females in a group $\left(g_{\mathrm{f}}\right)$, one-male versus many-males per group (categorical trait reflecting mating system), male versus female dispersal (categorical), dispersal rate of the dispersing sex, baseline mortality $\left(m_{\mathrm{b}}\right)$, disease-related mortality $\left(m_{\mathrm{i}}\right)$, incubation period $(b)$, and transmission probability $(\beta)$.

For comparative purposes, we also ran simulations under a non-spatial model, in which dispersing individuals can move to any group in the population after $d$ days of dispersal. These results provide additional insights into the importance of spatial structure in the spread of infectious disease.

\section{The spread of Ebola in African apes}

To investigate the dynamics of Ebola in African apes, we parameterized the spatial model using data from three populations of gorillas and four populations of chimpanzees (Table 2). The relative roles of host-to-host spread versus multiple independent transmissions of Ebola virus from a reservoir is uncertain (Walsh et al. 2003, 2005; Leroy et al. 2004; Pourrut et al. 2005), but it seems likely that at least some ape-to-ape transmission occurs within and possibly between groups (Caillaud et al. 2006; Bermejo et al. 2006; Walsh et al. 2007). Details on the host traits and sources of information are provided as footnotes to Table 2. Only females were allowed to disperse, and females left the group if the number of males dropped to zero $\left(T_{\mathrm{d}}=0\right)$. If all females died, a male remained in his range with no females, potentially attracting floater females. Dispersal was completed as quickly as 2 days $(d=2)$, provided that a group was located within that period of time.

Incubation of Ebola ranges from 2 to 30 days in humans. For these simulations, we used a value of 8 days, based on an estimate of incubation in a human who contracted the pathogen during necropsy of a dead chimpanzee at Taï Forest (Formenty et al. 1999b). Data on the duration of infection are unavailable for wild animals, and we required data on total duration for individuals that survived infection. We used an estimate of 14 days, based on data provided by Formenty et al. (1999b), in which the infected human was given medical treatment and survived. As in the other simulations, the probability of death followed a normal distribution, although we held $m_{\mathrm{i}}$ constant at 0.5 , giving a probability of death due to disease of $55.0 \%$ for infected-infectious individuals. To parameterize the probability of transmission $(\beta)$, we again used data from humans (Dowell et al. 1999). In $56 \%$ of households in which an Ebola outbreak occurred in this study, at least one secondary case occurred during a mean period of infection of 10 days, with $95 \%$ of the infections ending in death. Using the binomial theorem and the probability of escaping infection over a 10 -day period $(=44 \%)$, we calculated a per-day probability of infection of 0.079. We held this value constant in the simulations, although different strains of Ebola may cause different levels of mortality (Pourrut et al. 2005) and may also vary with regard to transmission rates. We used parameter settings for $m_{\mathrm{uf}}$ and $m_{\mathrm{if}}$ from the general simulations (Table 1). 


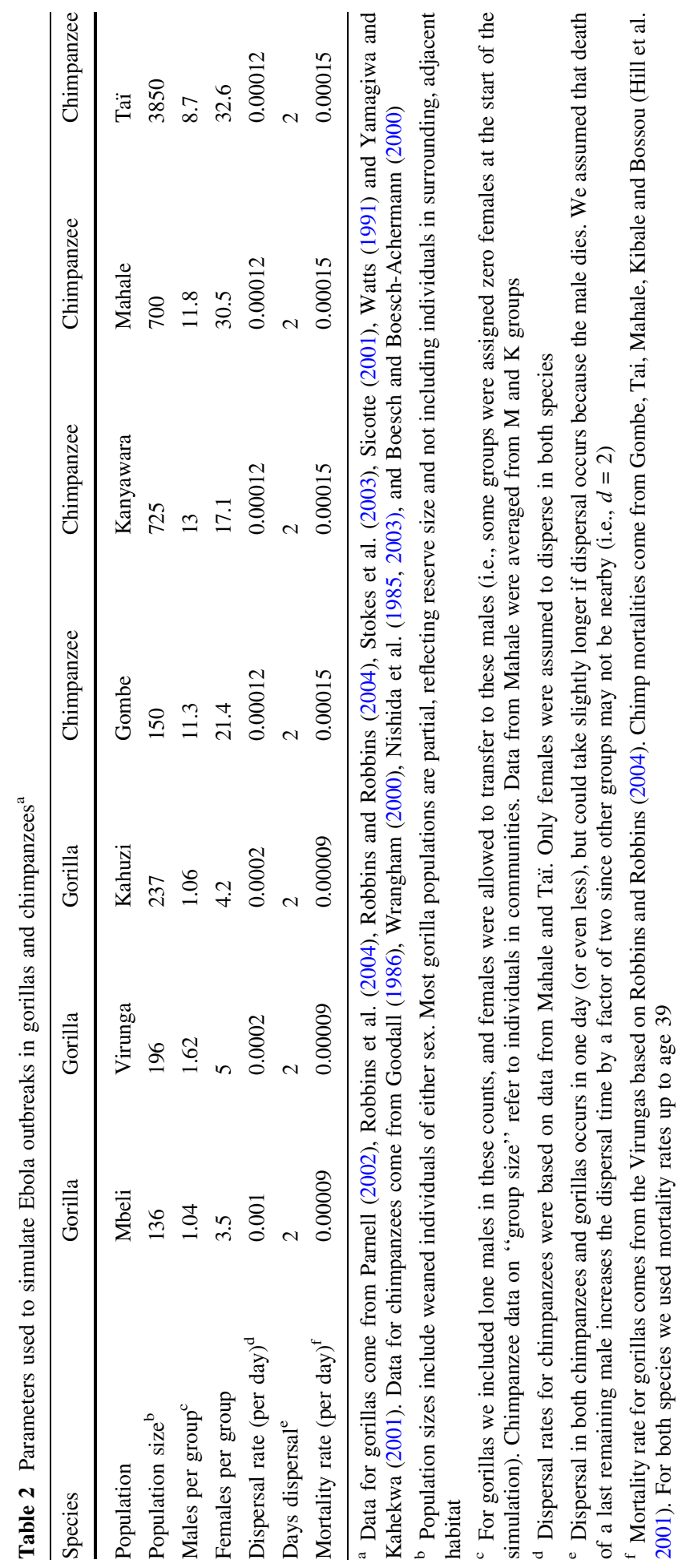




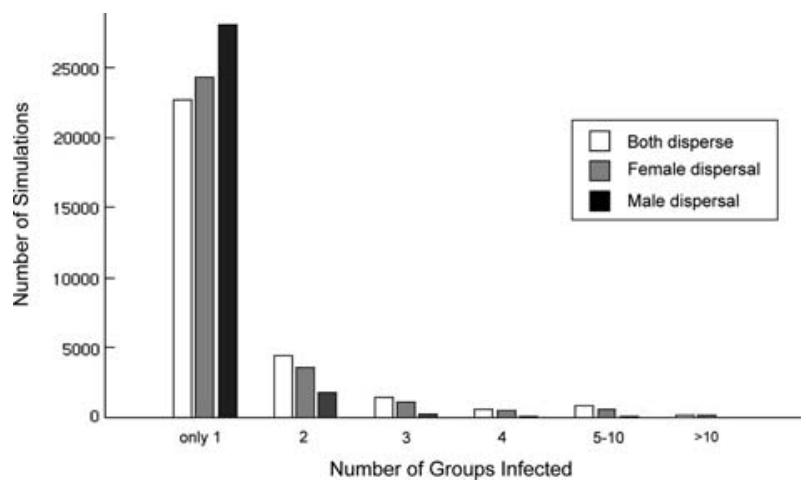

Fig. 2 Number of diseased groups in different runs of the simulation model. In most simulations, infection was present in only one group, with the disease rarely spreading to more than five groups, especially for male-only dispersal

\section{Results}

Simulations were initiated with infection of a single female and run until extinction of the pathogen in the simulated population. In most simulations, the disease was confined to a single social group and quickly disappeared from the population (Fig. 2), with the average simulation run lasting only 20.4 time steps (a time step is equivalent to 1 day; median $=18$ time steps, standard deviation $=11.2$ ). In nearly $3 \%$ of all simulations, however, over five social groups were infected following introduction of the disease. As shown in Fig. 2, more groups were infected when females or both sexes dispersed, as compared to male-only dispersal. In no cases did the host go extinct entirely, with a maximum loss of $53.9 \%$ of social groups. Thus, massive die-offs were relatively rare, suggesting that social substructuring of populations can buffer against disease outbreaks.
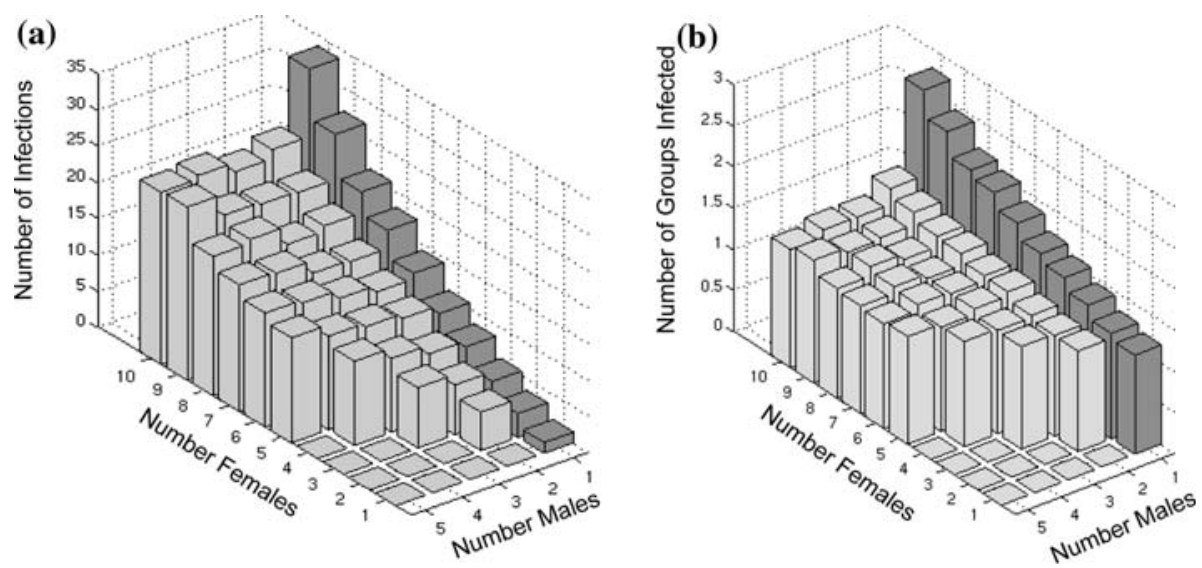

Fig. 3 Effect of disease in different social systems in a spatially explicit simulation model. Plots show (a) total number of individuals infected and (b) total number of groups infected. Simulations of a single-male systems are shown as dark bars (monogamy or polygyny), simulations of multi-male systems as light bars (polygynandry or monogamy). Both sexes dispersed at independent rates (Table 1) 
Table 3 Results from regression tree analyses

\begin{tabular}{|c|c|c|c|c|}
\hline $\begin{array}{l}\text { Dependent } \\
\text { variable }\end{array}$ & $\begin{array}{l}\text { Percentage } \\
\text { variation } \\
\text { explained }\end{array}$ & $\begin{array}{l}\text { Number } \\
\text { nodes }\end{array}$ & $\begin{array}{l}\text { Main variable (at } \\
\text { root of regression } \\
\text { tree) }\end{array}$ & $\begin{array}{l}\text { Other variables included in final regression } \\
\text { tree }\end{array}$ \\
\hline
\end{tabular}

Both sexes disperse in spatial model $(n=30,000)$

\begin{tabular}{|c|c|c|c|c|}
\hline $\begin{array}{l}\text { Number } \\
\text { infections }\end{array}$ & $15.2 \%$ & 6 & $\begin{array}{l}\text { Number } \\
\quad \text { females }>6.5\end{array}$ & $\begin{array}{l}\text { Longer incubation, more females, higher } \\
\text { summed dispersal rate, polygyny }\end{array}$ \\
\hline $\begin{array}{l}\text { Disease- } \\
\text { related } \\
\text { deaths }\end{array}$ & $22.7 \%$ & 9 & $\begin{array}{l}\text { Disease-related } \\
\text { mortality } \\
\left(m_{\mathrm{i}}\right)>0.26\end{array}$ & $\begin{array}{l}\text { More females, longer incubation, } \\
\text { polygyny, higher summed dispersal rate }\end{array}$ \\
\hline $\begin{array}{l}\text { Number of } \\
\text { groups } \\
\text { infected }\end{array}$ & $6.6 \%$ & 4 & Polygyny & $\begin{array}{l}\text { More females, lower disease-related } \\
\text { mortality, higher summed dispersal rate }\end{array}$ \\
\hline $\begin{array}{l}\text { Infected } \\
\text { dispersal }\end{array}$ & $10.0 \%$ & 2 & Polygyny & More females \\
\hline \multicolumn{5}{|c|}{ Either males or females disperse in spatial model $(n=60,000)$} \\
\hline $\begin{array}{l}\text { Number of } \\
\text { infections }\end{array}$ & $12.7 \%$ & 2 & $\begin{array}{l}\text { Number } \\
\quad \text { females }>5.5\end{array}$ & More females \\
\hline $\begin{array}{l}\text { Disease- } \\
\text { related } \\
\text { deaths }\end{array}$ & $26.9 \%$ & 11 & $\begin{array}{l}\text { Disease-induced } \\
\text { mortality } \\
\left(m_{\mathrm{i}}\right)>0.29\end{array}$ & $\begin{array}{l}\text { More females, greater dispersal, } \\
\text { polygynandry, longer incubation, higher } \\
\text { dispersal rate }\end{array}$ \\
\hline $\begin{array}{l}\text { Number of } \\
\text { groups } \\
\text { infected }\end{array}$ & $8.1 \%$ & 2 & Female dispersal & $\begin{array}{l}\text { Polygyny, more females, lower disease- } \\
\text { related mortality }\end{array}$ \\
\hline $\begin{array}{l}\text { Infected } \\
\text { dispersal }\end{array}$ & $13.9 \%$ & 3 & Female dispersal & Polygyny, more females \\
\hline
\end{tabular}

Notes: "Infected dispersal" refers to the number of individuals that disperse while infected, and could therefore carry the disease to other groups. "Other variables included..." are generally listed in their proximity to the root of the regression tree; this was not possible when a variable appeared at multiple levels

Graphical inspection of the data further revealed an effect of social system, with more infections in simulations of single-male groups (polygyny) and a striking effect of group size, indicated by increasing number of infections with increasing number of females in groups (Fig. 3a). The number of groups infected also increased in simulations of singlemale groups (Fig. 3b). As expected under the hypothesis of pathogen-mediated dispersal (PMD), this effect appeared to be driven by higher rates of female dispersal when the single male died from infection, resulting in increased transmission of the infection to neighboring groups - an effect that was less likely to occur in situations with more than one male in the group.

Results from the regression tree analyses are presented in Table 3. We first examined the effect of social system, life history traits and infection characteristics in simulations in which both sexes dispersed. Analyses using regression trees revealed that the number of infections in the population is primarily a function of the average number of females per group (Fig. 4a). The regression model obtained from this analysis predicts 10.2 infections when there are fewer than 6.5 females per group, and 20.9 infections when the average number of females is greater than 6.5. As shown in Fig. 4a, a longer incubation period, higher rates of dispersal, and simulation of single-male groups further increased the number of infections, with a mean of 50.6 infections in simulations of polygynous groups when the number of females $m_{\mathrm{f}}>6.5$, incubation period $b>6.5$, and the summed dispersal rate of males and females $\left(P_{\mathrm{md}}+P_{\mathrm{fd}}\right)>0.0067$. 

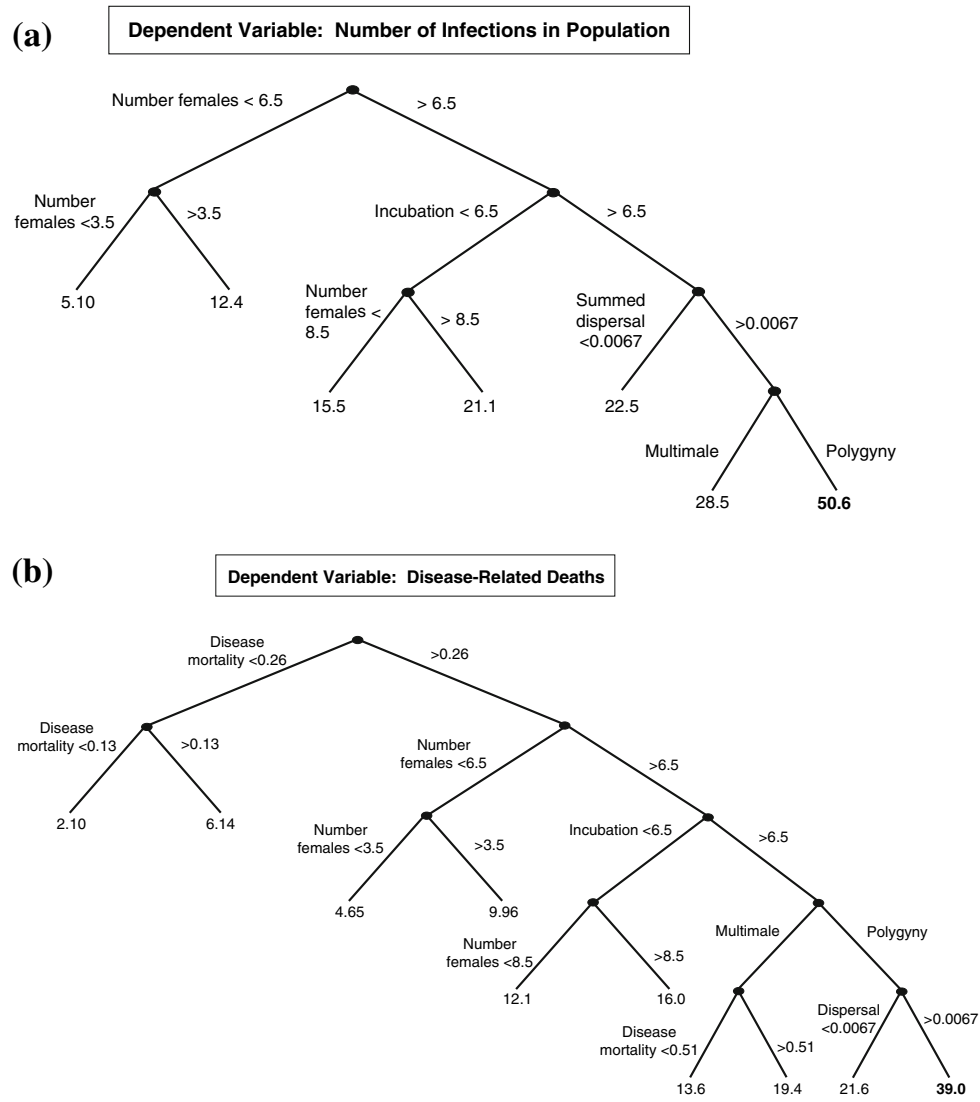

(c)

\section{Dependent Variable: Number of Groups Infected}

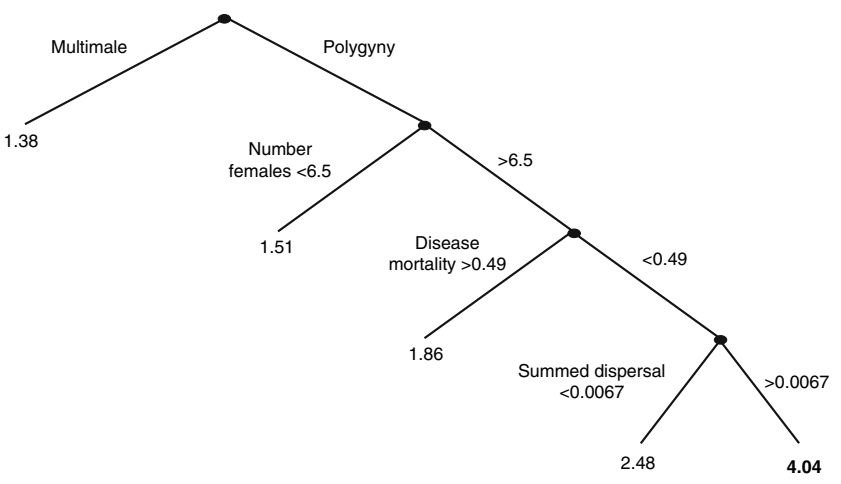

Fig. 4 Regresssion tree results when both sexes disperse (Analysis 1). Dependent variable in the regression tree was (a) total number of individuals infected, (b) disease-related deaths, and (c) number of groups infected. Regression trees make predictions for the hierarchical effect of predictor variables on a dependent variable. Trees are read by starting at the root at the top of the page, and following branches describing different values for predictor variables, some of which are nominal (see Methods). Labels show variables relevant for each node of the trees. Branches are arranged with the maximum predicted value indicated in bold at the far right of each tree 
Among the regression trees produced in Analysis 1, disease-related deaths accounted for the most variation in infection $(22.7 \%$, Table 3$)$. Not surprisingly, these analyses revealed that disease-related deaths were most tightly linked to disease-related mortality $\left(m_{\mathrm{i}}\right)$, which ranged from 0 to 1 and was used to calculate the per-day probability of death over the infectious period. The regression tree model predicted 4.2 deaths when $m_{\mathrm{i}}$ was less than 0.26 and 12.2 deaths for higher values of $m_{\mathrm{i}}$. Increases in the number of females, incubation period, polygyny and higher dispersal rates further increased mortality (Fig. 4b).

Group composition (polygyny) had a stronger impact on two dependent variables: number of groups infected and dispersal of infected individuals (Table 3). These analyses revealed that more groups were infected in simulations of single-male compared to multimale groups (Fig. 4c), with a further effect of the number of females in single-male groups (based on predictions from the regression model: 1.38 groups in multiple groups, 1.51 in single-male groups with fewer than 6.5 females, and 2.34 in single-male groups with more than 6.5 females). This pattern was driven by dispersal of infected individuals when the single male of a polygynous group died, with more instances of dispersal by infected individuals observed in simulations of single-male groups, particularly those with more females. However, the final regression tree was small (two nodes) and explained only $10 \%$ of the variation (Table 3 ).

In Analysis 2, we investigated the effect of female versus male dispersal by examining simulations in which only one sex dispersed $(n=60,000)$. In regression tree analyses, effects again depended on the dependent variable that was analyzed (Table 3). Thus, the number of infections increased with female group size, disease-related deaths increased with disease-related mortality, and number of groups infected increased in simulations of female-only dispersal. The model also predicted more dispersal events involving infected individuals under female dispersal, with a range of predictions from 1.17 to 6.98 when females dispersed, but only 0.23 when males dispersed (Fig. 5). These results involving number of groups infected and dispersal events differed from analyses of simulations when both sexes dispersed, where we found that polygyny (single-male groups) was the main variable at the root of the regression tree; however, polygyny was included in the tree at the second level in Analysis 2 (under female dispersal only, see Fig. 5). Finally, the regression tree analysis again accounted for the highest percentage of variance when examining

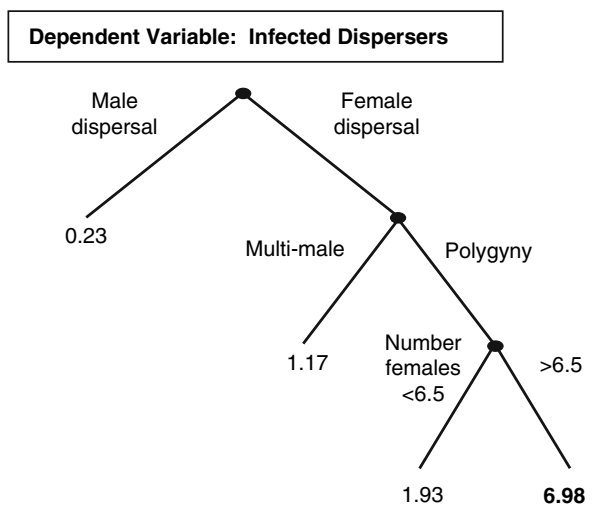

Fig. 5 Regression tree results for the number of infected dispersing individuals. The highest number of infected dispersers is predicted under female dispersal in polygynous systems with greater than six females per male. See legend for Fig. 4 on how to interpret this tree 
Table 4 Parameter settings for focused analyses of transmission probability and background mortality

\begin{tabular}{lllll}
\hline Variable & $\begin{array}{l}\text { Vary } \beta, \\
\text { one-male }\end{array}$ & $\begin{array}{l}\text { Vary } \beta, \\
\text { multi-male }\end{array}$ & $\begin{array}{l}\text { Vary } m_{\mathrm{b}}, \\
\text { one-male }\end{array}$ & $\begin{array}{l}\text { Vary } m_{\mathrm{b}}, \\
\text { multi-male }\end{array}$ \\
\hline Transmission probability $(\beta)$ & $0-0.08$ & $0-0.08$ & 0.4 & 0.4 \\
Baseline mortality rate per day $\left(m_{\mathrm{b}}\right)$ & 0.00002 & 0.00002 & $0-0.01$ & $0-0.01$ \\
Incubation period $(b)$ & 8 & 8 & 8 & 8 \\
Infectious period $(f)$ & 9 & 9 & 9 & 9 \\
Avg. number females per group $\left(g_{\mathrm{f}}\right)$ & 8 & 5 & 8 & 5 \\
Avg. number males per group $\left(g_{\mathrm{m}}\right)$ & 1 & 4 & 1 & 4 \\
Female dispersal rate $\left(P_{\mathrm{fd}}\right)$ & 0.005 & 0.005 & 0.005 & 0.005 \\
Male dispersal rate $\left(P_{\mathrm{md}}\right)$ & 0.002 & 0.002 & 0.002 & 0.002 \\
Disease-related mortality $\left(m_{\mathrm{i}}\right)$ & 0.55 & 0.55 & 0.55 & 0.55 \\
\hline
\end{tabular}

Note: For other parameter settings, see Table 1

disease-related deaths as a dependent variable, with $26.9 \%$ of the variance explained in the 11-node tree (not shown) that included both host traits and infection characteristics (Table 3).

Our analyses indicated that transmission probability $(\beta)$ and host baseline mortality $\left(m_{\mathrm{b}}\right)$ had little effect on patterns of infection. We therefore ran additional simulations to determine whether variation in these parameters had effects that were missed when other parameters were varied in the more general simulations. We set parameters as shown in Table 4, with 1,000 simulations run for each of the four conditions. For $\beta$, no significant effects were detected for number of infections or number of groups infected when other parameters were held constant. Instead, it appears that a lower transmission probability simply enables the disease to persist for more time steps (days) per simulation run, at least in polygynous groups (polygynous groups: $t_{999}=-4.37, P<0.001$; polygynandrous groups: $t_{999}=-1.24, P=0.22$ ); this did not appear to increase overall rates of infection through the dispersal of infected individuals. In the case of baseline mortality, further simulations also confirmed that there was no significant effect within the range of parameters described in Table 1. However, when allowed to range from 0 to 0.01 (one order of magnitude larger), we found that baseline mortality tended to increase rates of infection of individuals and groups (e.g., for number of infections in polygynandrous groups: $t_{999}=4.67, P<0.0001$; for number of groups infected in polygynandrous groups: $\left.t_{999}=3.82, P<0.0001\right)$. This was accompanied by significantly higher rates of male and female dispersal due to dissolution of groups.

Spatial versus non-spatial models of disease spread

The results presented above were derived from a spatially explicit model, with dispersing individuals following a random walk from their source group and thus tending to settle into nearby groups. We also ran simulations in which dispersal was unconstrained, with individuals moving to a randomly selected social group in the simulated population. As expected, this had a marked effect on the spread of disease, with much higher rates of disease spread and deaths (Table 5). It also amplified the effects of polygyny (Fig. 6). 
Table 5 Comparison of epidemiological patterns in spatial versus non-spatial simulations

\begin{tabular}{lccc}
\hline Variable & Spatial: mean (SD) & Non-spatial: mean (SD) & $F$-statistic \\
\hline Number infections & $15.6(18.0)$ & $36.0(162)$ & 470 \\
Percentage groups lost & $1.49(1.38)$ & $3.19(12.9)$ & 270 \\
Disease-related deaths & $10.1(12.2)$ & $21.9(102)$ & 394 \\
Infected dispersal & $2.35(6.02)$ & $7.82(50.2)$ & 352 \\
\hline
\end{tabular}

Note: Table shows means with standard deviations (SD) in parentheses and F-statistics based on 60,000 simulation runs in which both sexes could disperse. All results were significant at $P<0.0001$
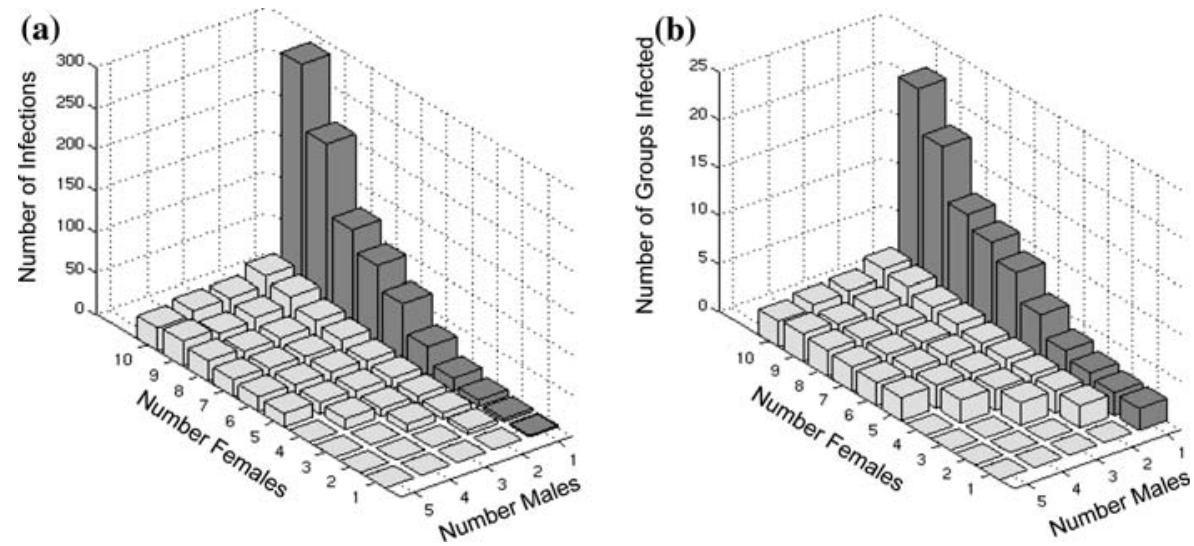

Fig. 6 Effect of disease in different social systems in a non-spatial model (cf. Fig. 3). Plots show (a) total number of individuals infected and (b) total number of groups infected. Simulations of a single-male (polygynous) system are shown as dark bars, simulations of non-polygynous systems as light bars. Both sexes dispersed at independent rates (Table 1)

The spread of Ebola in African apes

We parameterized the model using data from seven African ape populations (Table 2) and data on infection characteristics of Ebola (see Methods). In comparing patterns of infection and death for gorillas and chimpanzees (Fig. 7), results indicate a profound effect of social system (infection characteristics were held constant in these simulations). Following introduction of a single infection, the number of diseased individuals (Fig. 7a) and number of deaths (Fig. 7b) was higher in the larger multi-male multi-female chimpanzee groups than in the smaller gorilla groups that more typically have a single male. In gorillas, a larger number of infected individuals dispersed (Fig. 7c) and more groups were infected (Fig. 7d). The largest number of infections and deaths due to disease were found in the Virungas gorilla population, despite the larger number of males (on average) in these groups. However, the Virungas groups also have more females per group, which we found to be a key driver for the number of infections in the more general simulations (Fig. 4a). Among chimpanzees, more infections and deaths occurred in simulations of the Taï population, probably reflecting both large group sizes and a large estimated population size. 

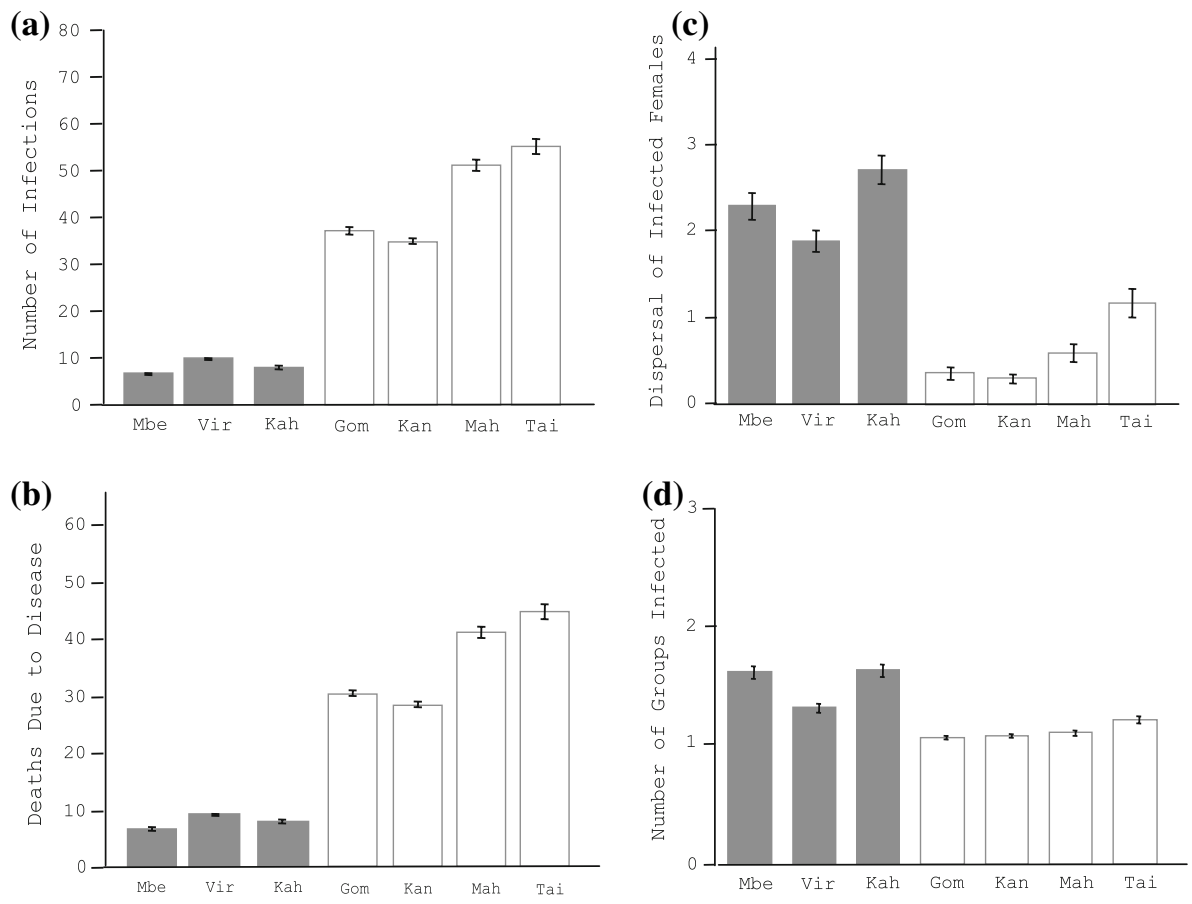

Fig. 7 Simulations parameterized for two African apes. Plots show results (mean values $+1 \mathrm{SE}$ ) for (a) number of infections, (b) deaths due to disease, (c) dispersal of infected females, and (d) number of groups infected. Filled bars: gorillas; open bars: chimpanzees. Population codes: Mbe: Mbeli; Vir: Virungas; Kah: Kahuzi; Gom: Gombe; Kan: Kanyawara; Mah: Mahale; Taï: Taï. See Table 2 and text for details on model parameterization

\section{Discussion}

We investigated the links between disease spread, host social systems and infection characteristics in a spatially explicit, individual-based model. We found that group composition, dispersal patterns and disease characteristics played interacting roles in accounting for population-level impacts following introduction of a highly virulent pathogen. Moreover, different parameters had different influences on the spread of disease, with number of females (a measure of group size) impacting the number of infections in the population, and mating system impacting group-to-group spread. For the latter, singlemale systems tended to result in a larger number of groups being infected, especially when single-male groups contained more than six females. These results therefore lend theoretical support to the hypothesis of PMD. Specifically, a highly pathogenic disease can cause higher rates of dispersal when the male of a polygynous group dies from disease, with females moving to new groups and potentially carrying the disease with them; because females are the source of infection other groups, the population-level effects of PMD should increase as the number of females in the group increases. This effect contrasts with the usual expectation that disease-related deaths reduce the spread of disease, for example by reducing values of $R_{0}$ (the basic reproductive number that determines the success of parasite colonization, see Anderson and May 1979, 1991). 
A number of other host traits and infection characteristics had an impact on the spread of disease in the simulations, including incubation period, rates of dispersal, and diseaserelated mortality. Importantly, the regression tree analyses provided new insights by suggesting that some parameters had effects only when other parameters reached a threshold value. For example, incubation period had a major effect on the number of infections observed, but only when the number of females per group was relatively large ( $>6.5$, see Fig. 4a). Dispersal rate also had an effect, but only when incubation period itself was greater than 6.5 days. As a final example, when comparing male and female dispersal, evidence for PMD as a significant force emerged only when females dispersed-as expected given that PMD relies on female dispersal under the social parameters used herewith additional variance explained by the number of females in single-male (but not in multi-male) groups (Fig. 5). Thus, the regression tree analyses revealed strikingly hierarchical effects and proved very useful for analyzing the simulation output.

Our analyses also indicated that several of the traits included in our model had relatively little impact on disease spread; these included one host trait (baseline mortality, $m_{\mathrm{b}}$ ) and one infection parameter (transmission probability, $\beta$ ). The absence of an effect of baseline mortality probably reflects that the potential for epidemic spread of a virulent pathogen following initial introduction is more dependent on disease-related mortality than baseline mortality (Anderson and May 1991). In fact, the average duration of the simulation in the spatially explicit model was only 20.4 days-the time period from initiation of the infection in one individual to extinction of the pathogen in the simulated population. Over this short time span, the baseline mortality rate $m_{\mathrm{b}}$ of $0-0.001$ was unlikely to have a major effect. When this value of mortality was expanded to 0.01 , mortality began to have an effect, with increased rates of mortality leading to more infections and apparently driven by increased dispersal of infected individuals (both males and females).

It is more challenging to understand why the transmission coefficient $\beta$ had no significant effect in the statistical analyses, as it is a key variable in epidemiological models (Anderson and May 1979, 1991). This may reflect that even at low per-contact transmission rates, the disease quickly spreads to most members of a group, well within the duration of infection, or that equations that use $\beta$, such as $R_{0}$, are simply less relevant to understanding parasite establishment in highly socially structured populations (Cross et al. 2005). In addition, given the high value of mortality in this study, which was used to characterize real-world cases of emerging diseases, it is possible that the disease will always die out regardless of the value of $\beta$. Finally, it could be that $\beta$ has non-linear effects that were not detected in the regression tree analyses. However, follow-up simulations described in the Results revealed no such effects.

We also investigated the effects of spatial structure by contrasting results from a model in which dispersal was distance-dependent (spatially explicit) to one in which individuals could move to any group in the simulated population with equal probability (non-spatial). As expected, the overall impact of an introduced pathogen was lessened when individuals dispersed locally, with most movements to nearby groups. In this case, multiple infected dispersers were probably more likely to enter the same nearby group, effectively limiting the spread of disease. In addition, disease tended to reduce the local density of groups once an epidemic was underway, leading to longer dispersal times and either death or recovery of infected individuals before they located a new group.

Our finding that PMD can increase the spread of disease raises two questions: is this a case of selection on pathogen virulence to manipulate host dispersal, and could this mechanism select for host behaviors that limit the spread of disease? Parasite induced behaviors are expected to provide benefits to the pathogen in the form of increased 
transmission (Dobson 1988; Moore 2002). However, under PMD, the benefits would arise from increasing the rate of disease-related mortality, which in most models should act to decrease $R_{0}$ (Anderson and May 1991). Thus, mortality would be expected to provide benefits and costs to the pathogen, with the benefits involving increased dispersal in some social settings, and the costs involving increased mortality. The net benefits are likely to be positive only when groups have relatively large numbers of females, and when incubation period relative to dispersal time is large. In terms of host behavioral defenses, we might expect that both males and females more vigorously prevent immigration in polygynous systems. Presently, no data are available to test this prediction.

In primates, the number of males in a social group tends to correlate with the number of females (Andelman 1986; Altmann 1990, 1999; Mitani et al. 1996; Nunn 1999; Lindenfors et al. 2004). Our process of group formation generated such an association when initializing the simulated populations. The most female-biased sex ratio in our simulations was 10 females for one male, a ratio that is common in many primate populations. In the comparative database of 49 primate species used by Mitani et al. (1996), for example, three polygynous primate species have values approximating or exceeding this expected ratio (Cercopithecus ascanius: 9.5 females per male; Cercopithecus mitis: 18 females per male; Presbytis entellus: 12 females per male). Moreover, several gorilla populations contain groups that exceed this ratio (Kalpers et al. 2003; Robbins and Robbins 2004; Robbins et al. 2004). Thus, despite a general association between the number of males and number of females, substantial variation exists, and the simulated values are within the range of natural variation, indicating that PMD has the potential to occur in species of primates characterized by polygynous mating systems.

Even if a pathogenic disease cannot be sustained in a single host population for a long period of time, the simulations revealed that the impacts of an introduced pathogen could be substantial in terms of population impacts. Moreover, many emerging diseases of wildlife are initiated through repeated cross-species transmission ("spillover") from a reservoir host (Daszak et al. 2000; Gog et al. 2002; Nunn and Altizer 2006). These repeated introductions could maintain the infection for a longer period of time, producing greater population declines. Finally, the widespread declines that can follow pathogen introduction highlight the need for greater preparedness to deal with emerging diseases in threatened populations, including the need for regular monitoring of mortality and pathogens, veterinary staff to deal with outbreaks, and disease-related planning based on models that explicitly incorporate epidemiologically relevant variation in social and mating behavior.

Future research could examine additional dimensions of the model that we developed, along with variations in social systems that might impact the spread of disease. Particularly relevant in terms of PMD are details on the dispersal process. For example, we assumed that floaters were effectively isolated, even when individuals dispersed simultaneously from the same group, or when floaters from different groups overlapped in the same grid cell; infections in the floater pool could therefore not spread until an infected individual entered a social group. In many primate species, however, floater individuals live in groups (Pusey and Packer 1987) in which socially transmitted diseases can spread. Thus, we might predict that the existence of such groups would greatly facilitate the spread of diseases, even when dispersal time is long relative to incubation period. It would also be interesting to include age structure, given that many dispersing individuals are likely to be immature, could have under-developed immune systems, and often have different patterns of association within social groups. Finally, in some species, females might not always disperse to new groups after the male dies, with satellite males instead joining the group of females. 
The simulation results show that host social system, life history and dispersal have a synergistic effect on the spread of a virulent, directly transmitted pathogen in a population of susceptible individuals. Previous theoretical research has revealed similar effects for other types of pathogens. Using a non-spatial individual-based simulation model to examine the spread of STDs in polygynous systems, for example, Thrall et al. (2000) found a non-linear relationship between host mortality and equilibrium disease prevalence. When mortality was low, increases in host death rates led to higher dispersal, thus increasing overall disease prevalence. However, for high host mortality rates, further increases resulted in a shorter duration of the infectious period and a decline in population disease prevalence, thus producing a non-monotonic relationship between mortality and disease prevalence. This is analogous to the impact of predators on host-pathogen dynamics in which low levels of predation can result in increased disease prevalence due to the greater recruitment of susceptible hosts into the population. However, further increases in predation results in lower prevalence due to negative impacts on the duration of infection (Holt 2005).

Models of disease spread commonly focus on $R_{0}$ (Anderson and May 1979, 1991). However, standard formulations of $R_{0}$ do not take into account the structuring of host populations into social groups of different size and composition, or the distribution of these groups in time and space (Hess 1996a; Thrall et al. 2000; Cross et al. 2005). In some sense, this situation is analogous to models that have explored the effects of connectivity and habitat fragmentation on disease dynamics. Thus, Hess (1996a, b) developed an island metapopulation model which considers both within and among-patch dynamics. In general, he found that increased connectivity among habitat patches led to a greater likelihood of the epidemic spread of contagious diseases through the system, with increased potential for overall extinction. However, Gog et al. (2002) showed that if there are alternative hosts and thus the possibility of "disease spillover" onto the focal species (a common concern when emerging epidemic diseases are involved), then increasing among-patch migration (above some threshold level) may have the net positive effect of increasing the fraction of sites occupied. Potentially this could also apply to populations with spatially sub-structured social or mating groups where multiple hosts are involved.

While these models (including the one presented here) demonstrate that among-group or patch movement can impact disease spread and extinction risk, a major simplification is the absence of genetic considerations (i.e., host resistance). While this assumption may be acceptable for some newly emerged or exotic diseases (e.g., chestnut blight in North America), there are an appreciable number of exceptions to this generalization, and it is unlikely to apply to all pathogens. Following on the study by Hess (1996a), McCallum and Dobson (2002) showed analytically that when a resistant class of hosts was present, then despite the fact that the fraction of infected patches increased with greater among-patch movement, total occupancy (and the fraction of resistant patches) also increased, permitting susceptible hosts to persist. As another example, Carlsson-Granér and Thrall (2002) used a spatially explicit metapopulation approach to demonstrate that the evolution of plant host resistance, and therefore observed levels of disease incidence and prevalence are likely to be critically affected by the degree of population aggregation (conceptually equivalent to levels of among-group migration in animals). In particular, they found that with increasing among-population connectivity, host resistance evolved more rapidly to higher levels than did disease incidence (fraction of sites with disease present). However, due to higher average levels of resistance, there was a negative correlation between connectivity and disease prevalence (fraction of individuals infected per site). Thus, host persistence was actually greater in less-fragmented situations. 
Disease, mating systems, and conservation of Great Apes

Simulations parameterized with data on gorillas and chimpanzees further highlighted the inter-relationships between group size, pathogen virulence and dispersal of individuals between groups. The results also have implications for conservation of African apes and other mammals, and a similar approach could be implemented in a more formal population viability analysis (PVA). Previous simulations of population dynamics of realistically socially structured gorilla populations have been conducted (Akçakaya and Ginzburg 1991; Durant 2000). Both analyses indicated relatively strong probability of persistence over centuries of either the population, or of genetic heterogeneity in the population. However, disease and its transmission by dispersing individuals were not incorporated in these simulations. More recent modeling of the spread of Ebola in gorillas has been conducted using data on Ebola infections in real populations. Caillaud et al. (2005) found that it was not possible to determine whether spread occurred through spillover from a reservoir or through ape-to-ape transmission, and they documented that infection rates were significantly higher among animals living in groups. Based on patterns of disease spread among gorilla groups relative to the typical Ebola disease cycle, Bermejo et al. (2006) concluded that ape-to-ape transmission was a likely explanation for the spread of Ebola in the Lossi sanctuary in the Republic of Congo. Walsh et al. (2007) consider the mechanisms by which inter-group transmission of Ebola could occur in apes, which previous authors concluded was likely to be rare (Tutin 2000).

Previous analyses of gorilla population dynamics were based on data from mountain gorilla populations, of which almost half the social groups are multi-male (Robbins 2001; Kalpers et al. 2003). About $90 \%$ of the world's gorillas live in West Africa, where over 90\% of groups are single-male (Parnell 2002; Robbins et al. 2004). These previous studies indicated a strong positive effect of dispersal on persistence, which implies that the western gorilla populations would be even more persistent in the absence of disease than mountain gorillas. By contrast, the adverse effect of disease-related male mortality on dispersal of infected females in a polygynous system indicates a radically different outcome. Hess $(1996 a, b)$ simulated a similar effect of disease, also assuming a completely susceptible host population, and showed that under certain combinations of infection probability and disease-induced mortality, dispersal resulted in reduced population size through transmission to additional sub-populations (although dispersal could also play a role in generating genetic variation needed to maintain resistance to disease). The reality of diseased individuals as lethal carriers of disease is amply illustrated by the death of a whole group of hyraxes (Procavia johnstoni) in Serengeti National Park, Tanzania, following immigration of a single individual infected with mange (Hoeck 1982; see Barrett and Henzi 1998 for a different example in baboons).

In terms of conservation action, our results suggest that different control measures will be needed for different social systems, and this point is brought home more clearly by comparing the expected spread of disease in gorillas and chimpanzees. The species to target for disease control will depend on whether it is more important to preserve the number of groups or the total number of individuals; in the former case, efforts should focus on combating disease in polygynous gorillas, while in the latter, a larger number of individuals in a chimpanzee community are likely to die in a disease outbreak (at least under the assumptions of equal contact probabilities used here). Choices in this regard will depend on details of the behavior and status of the host in an area, including the number of groups that remain, the distribution of genetic variation within versus between groups, and how changes in group size and composition impact population growth. For example, a 
reduction in group size or dissolution of groups via male death could increase infanticide risk, depending on whether males protect infants against infanticide, and whether females disperse to new groups with dependent offsrping.

In conclusion, the results presented here show how individual-based models can be used to investigate the complex interactions that exist between host traits, infection characteristics, and spatial structure in real-world host-pathogen systems. We used this model to investigate the conditions under which pathogenic diseases can spread through increased dispersal of individuals, specifically when one sex dies out in a group. As an example, we applied the model to investigate the spread of Ebola - a highly pathogenic disease - in African apes. Because many emerging pathogens of wildlife, including Ebola, can be transmitted from wildlife to humans, understanding the host traits that most effectively maintain pathogens in wildlife also could be important for identifying and mitigating disease-related threats to human health.

Acknowledgements We thank Derek Roff, Paul Cross, Martha Robbins, Andrew Robbins, Sonia Altizer and two anonymous reviewers for helpful suggestions and discussion. This research was supported by the NSF (\#DEB-0212096 to CN), The Max Planck Society, Conservation International, and the National Center for Ecological Analysis and Synthesis (NCEAS).

\section{References}

Akçakaya HR, Ginzburg LR (1991) Ecological risk analysis for single and multiple populations. In: Seitz A, Loeschke V (eds) Species conservation: a population-biological approach. Birkhaüser Verlag, Basel, pp 73-87

Alexander KA, Appel MJG (1994) African wild dogs (Lycaon pictus) endangered by a canine-distemper epizootic among domestic dogs near the Masai-Mara national reserve, Kenya. J Wildlife Dis 30:481-485

Altizer S, Nunn CL, Thrall PH, Gittleman JL, Antonovics J, Cunningham AA, Dobson AP, Ezenwa V, Pedersen AB, Poss M, Pulliam JRC (2003) Social organization and parasite risk in mammals: integrating theory and empirical studies. Annu Rev Ecol Evol Syst 34:517-547

Altmann J (1990) Primate males go where the females are. Anim Behav 39:193-195

Altmann J (1999) Models of outcome and process: predicting the number of males in primate groups. In: Kappeler PM (ed) Primate Males. Cambridge University Press, Cambridge, pp 236-247

Andelman SJ (1986) Ecological and social determinants of cercopithecine mating patterns. In: Rubenstein DI, Wrangham RW (eds) Ecological aspects of social evolution: birds and mammals. Princeton University Press, Princeton, NJ, pp 201-216

Anderson RM, May RM (1979) Population biology of infectious diseases: part 1. Nature 280:361-367

Anderson RM, May RM (1981) The population-dynamics of micro-parasites and their invertebrate hosts. Philos Trans Roy Soc Lond Ser B Biol Sci 291:451-524

Anderson RM, May RM (1991) Infectious diseases of humans: dynamics and control. Oxford University Press

Appel MJG, Summers BA (1995) Pathogenicity of morbilliviruses for terrestrial carnivores. Veterinary Microbiology 44:187-191

Barrett L, Henzi SP (1998) Epidemic deaths in a chacma baboon population. S Afr J Med Sci 94:441

Berger L, Speare R, Daszak P, Green DE, Cunningham AA, Goggin CL, Slocombe R, Ragan MA, Hyatt AD, McDonald KR, Hines HB, Lips KR, Marantelli G, Parkes H (1998) Chytridiomycosis causes amphibian mortality associated with population declines in the rain forests of Australia and Central America. Proc Natl Acad Sci USA 95:9031-9036

Bermejo M, Rodriguez-Teijerio JD, Illera G, Barroso A, Vila C, Walsh PD (2006) Ebola outbreak killed 5000 gorillas. Science 314:1564

Boesch C, Boesch-Achermann H (2000) The chimpanzees of the Taï Forest. Oxford University Press, Oxford

Boots M, Sasaki A (1999) 'Small worlds' and the evolution of virulence: infection occurs locally and at a distance. Proc Roy Soc Lond Ser B Biol Sci 266:1933-1938 
Caillaud D, Levréro F, Cristescu R, Gatti S, Dewas M, Douadi M, Gautier-Hion A, Raymond M, Menard N (2006) Gorilla susceptibility to Ebola virus: the cost of sociality. Curr Biol 16:R489-R491

Carlsson-Granér U, Thrall PH (2002) The spatial distribution of plant populations, disease dynamics and evolution of resistance. Oikos 97:97-110

Carpenter MA, Appel MJG, Roelke-Parker ME, Munson L, Hofer H, East M, O’Brien SJ (1998) Genetic characterization of canine distemper virus in Serengeti carnivores. Veter Immunol Immunopathol 65:259-266

Cleaveland S, Hess GR, Dobson AP, Laurenson MK, McCallum HI (2002) The role of pathogens in biological conservation. In: Hudson PJ, Rizzoli A, Grenfell BT, Heesterbeek H, Dobson AP (eds) The ecology of wildlife diseases. Oxford University Press, New York, pp 139-150

Cross PC, Lloyd-Smith JO, Johnson PL, Getz WM (2005) Dueling time scales of host movement and disease recovery determine invasion of disease in structured populations. Ecol Lett 8:587-595

Daszak P, Berger L, Cunningham AA, Hyatt AD, Green DE, Speare R (1999) Emerging infectious diseases and amphibian population declines. Emerg Infect Dis 5:735-748

Daszak P, Cunningham AA, Hyatt AD (2000) Emerging infectious diseases of wildlife: threats to biodiversity and human health. Science 287:443-449

Daszak P, Cunningham AA, Hyatt AD (2003) Infectious disease and amphibian population declines. Div Distrib 9:141-150

De'ath G, Fabricius KE (2000) Classification and regression trees: a powerful yet simple technique for ecological data analysis. Ecology 81:3178-3192

Dobson AP (1988) The population biology of parasite-induced changes in host behavior. Quart Rev Biol 63:139-165

Dobson A, Foufopoulos J (2001) Emerging infectious pathogens of wildlife. Philos Trans Roy Soc Lond Ser B Biol Sci 356:1001-1012

Dowell SF, Mukunu R, Ksiazek TG, Khan AS, Rollin PE, Peters CJ (1999) Transmission of Ebola hemorrhagic fever: a study of risk factors in family members, Kikwit, Democratic Republic of the Congo, 1995. J Infect Dis 179:S87-S91

Durant SM (2000) Dispersal patterns, social organization and population viability. In: Gosling LM, Sutherland WJ (eds) Behaviour and conservation. Cambridge University Press, Cambridge, pp 172-197

Formenty P, Boesch C, Wyers M, Steiner C, Donati F, Dind F, Walker F, Le Guenno B (1999a) Ebola virus outbreak among wild chimpanzees living in a rain forest of Cote d'Ivoire. J Infect Dis 179:S120-S126

Formenty P, Hatz C, Le Guenno B, Stoll A, Rogenmoser P, Widmer A. (1999b) Human infection due to Ebola virus, subtype Cote d'Ivoire: clinical and biologic presentation. J Infect Dis 179:S48-S53

Funk SM, Fiorello CV, Cleaveland S, Laurenson K, Gompper ME (2001) The importance of disease in carnivore conservation. Pages 11-34 In: Gittleman JL, Funk S, Macdonald DW, Wayne RK (eds) Carnivore conservation. Cambridge University Press, Cambridge, pp 11-34

Gandon S, Capowiez Y, Dubois Y, Michalakis Y, Olivieri I (1996) Local adaptation and gene-for-gene coevolution in a metapopulation model. Proc Roy Soc Lond Ser B Biol Sci 263:1003-1009

Gog J, Woodroffe R, Swinton J (2002) Disease in endangered metapopulations: the importance of alternative hosts. Proc Roy Soc Lond Ser B Biol Sci 269:671-676

Goodall J (1986) The chimpanzees of Gombe: patterns of behavior. Harvard University Press, Cambridge, MA

Hess G (1996a) Disease in metapopulation models: implications for conservation. Ecology 77:1617-1632

Hess G (1996b) Linking extinction to connectivity and habitat destruction in metapopulation models. Am Nat 148:226-236

Hill K, Boesch C, Goodall J, Pusey A, Williams J, .Wrangham R (2001) Mortality rates among wild chimpanzees. J Human Evol 40:437-450

Hoeck HN (1982) Population dynamics, dispersal and genetic isolation in two species of hyrax (Heterohyrax brucei and Procavia johnstoni) on habitat islands in the Serengeti. Z Tierpsychol 59:177-210

Holt RD (2005) The community context of disease emergence: could changes in predation be a key driver? In: Ostfeld R, Keesing F, Eviner V (eds) Infectious disease ecology: effects of disease on ecosystems and of ecosystems on disease. Princeton University Press (in Press)

Kalpers J, Williamson EA, Robbins MM, McNeilage A,Nzamurambaho A, Ndakasi L, Mugiri G (2003) Gorillas in the crossfire: population dynamics of the Virunga mountain gorillas over the past three decades. Oryx 37:326-337

Kennedy S, Kuiken T, Jepson PD, Deaville R, Forsyth M, Barrett T, van de Bildt MWG, Osterhaus ADME, Eybatov T, Duck C, Kydyrmanov A, Mitrofanov I, Wilson S (2000) Mass die-off of Caspian seals caused by canine distemper virus. Emerg Infect Dis 6:637-639

Kock RA, Wambua JM, Mwanzia J Wamwayi H, Ndungu EK, Barrett T, Kock ND, Rossiter PB (1999) Rinderpest epidemic in wild ruminants in Kenya 1993-97. Veter Record 145:275-283 
Leroy EM, Rouquet P, Formenty P, Souquiere S, Kilbourne A, Froment JM, Bermejo M, Smit S, Karesh W, Swanepoel R, Zaki SR, Rollin PE (2004) Multiple Ebola virus transmission events and rapid decline of central African wildlife. Science 303:387-390

Lindenfors P, Froberg L, Nunn CL (2004) Females drive primate social evolution. Proc Roy Soc Lond Ser B Biol Sci 271(Suppl):S101-S103

Lockhart AB, Thrall PH, Antonovics J (1996) Sexually transmitted diseases in animals: ecological and evolutionary implications. Biol Rev Cambridge Philos Soc 71:415-471

Loehle C (1995) Social barriers to pathogen transmission in wild animal populations. Ecology 76:326-335

May RM, Anderson RM (1979) Population biology of infectious diseases: part II. Nature 280:455-461

McCallum H, Dobson A (2002) Disease, habitat fragmentation and conservation. Proc Roy Soc Lond Ser B Biol Sci 269:2041-2049

Mitani JC, Gros-Louis J, Manson JH (1996) Number of males in primate groups: comparative tests of competing hypotheses. Am J Primatol 38:315-332

Moore J (2002) Parasites and the behavior of animals. Oxford University Press, Oxford

Morse SS (1995) Factors in the emergence of infectious-diseases. Emerg Infect Dis 1:7-15

Nishida T, Hiraiwa-Hasegawa M, Hasegawa T, Takahata Y (1985) Group extinction and female transfer in wild chimpanzees in the Mahale National Park, Tanzania. Z Tierpsychol 67:284-301

Nishida T, Corp N, Hamai M, Hasegawa T, Hirawa-Hasegawa M, Hosaka K, Hunt KD, Itoh N, Kawanaka K, Matsumoto-Oda A, Mitani JC, Nakamura M, Norikoshi K, Sakamaki T, Turner L, Uehara S, Zamma K (2003) Demography, female life history, and reproductive profiles among the chimpanzees of Mahale. Am J Primatol 59:99-121

Nunn CL(1999) The number of males in primate social groups: a comparative test of the socioecological model. Behav Ecol Sociobiol 46:1-13

Nunn CL, Altizer SM (2006) Infectious diseases in primates: behavior, ecology and evolution. Oxford University Press, Oxford

O'Keefe KJ, Antonovics J (2002) Playing by different rules: the evolution of virulence in sterilizing pathogens. Am Nat 159:597-605

Osterhaus ADME, Deswart RL, Vos HW, Ross PS, Kenter MJH, Barrett T (1995) Morbillivirus infections of aquatic mammals - newly identified members of the genus. Veter Microbiol 44:219-227

Parnell RJ (2002) Group size and structure in Western Lowland Gorillas (Gorilla gorilla gorilla) at Mbeli Bai, Republic of Congo. Am J Primatol 56:193-206

Pourrut X, Kumulungui B, Wittmann T, Moussavou G, Délicat A, Yaba P, Nkoghe D, Gonzalez JP, Leroy EM (2005) The natural history of Ebola virus in Africa. Microbes Infect 7:1005-1014

Pusey AE, Packer C (1987) Dispersal and philopatry. In: Smuts BB, Cheney DL, Seyfarth RM, Wrangham RW, Struhsaker TT (eds) Primate societies. University of Chicago Press, Chicago, pp 250-266

Reichard UH (2003) Social monogamy in gibbons: the male perspective. In: Reichard UH, Boesch C (eds) Monogamy: mating strategies and partnerships in birds, humans and other mammals. Cambridge University Press, Cambridge, pp 190-213

Robbins MM (2001) Variation in the social system of mountain gorillas: the male perspective. In: Robbins MM, Sicotte P, Stewart KJ (eds) Mountain gorillas: three decades of research at karisoke. Cambridge University Press, Cambridge, pp 29-58

Robbins MM, Bermejo M, Cipolletta C, Magliocca F, Parnell RJ, Stokes E (2004) Social structure and lifehistory patterns in western gorillas (Gorilla gorilla gorilla). Am J Primatol 64:145-159

Robbins MM, a Robbins AM (2004) Simulation of the population dynamics and social structure of the Virunga mountain gorillas. Am J Primatol 63:201-223

Roelke-Parker ME, Munson L, Packer C, Kock R, Cleaveland S, Carpenter M, O'Brien SJ, Pospischil A, Hofmann-Lehmann R, Lutz H, Mwamengele GLM, Mgasa MN, Machange GA, Summers BA, Appel MJG (1996) A canine distemper virus epidemic in Serengeti lions (Panthera leo). Nature 379:441-445

Roff DA, Roff RJ (2003) Of rats and Maoris: a novel method for the analysis of patterns of extinction in the New Zealand avifauna before European contact. Evol Ecol Res 5:759-779

Rossiter P (2001) Morbilliviral diseases. In: Williams ES, Barker IK (eds) Infectious diseases of wild mammals. Iowa State University Press, Ames, Iowa, pp 37-45

Roy BA, Kirchner JW (2000) Evolutionary dynamics of pathogen resistance and tolerance. Evolution 54:51-63

Sicotte P (2001) Female mate choice in mountain gorillas. In: Robbins MM, Sicotte P, Stewart KJ (eds) Mountain gorillas: three decades of research at Karisoke. Cambridge University Press, Cambridge, pp $59-87$

Slatkin M, Hausfater G (1976) A note on the activities of a solitary male baboon. Primates 17:311-322

Stokes EJ, Parnell RJ, Olejniczak C (2003) Female dispersal and reproductive success in wild western lowland gorillas (Gorilla gorilla gorilla). Behav Ecol Sociobiol 54:329-339 
Thrall PH, Antonovics J (1995) Theoretical and empirical-studies of metapopulations-population and genetic dynamics of the Silene-Ustilago system. Can J Bot Revue Canadienne De Botanique 73:S1249-S1258

Thrall PH, Antonovics J, Dobson AP (2000) Sexually transmitted diseases in polygynous mating systems: prevalence and impact on reproductive success. Proc Roy Soc Lond B 267:1555-1563

Thrall PH, Antonovics J, Wilson WG (1998) Allocation to sexual versus nonsexual disease transmission. Amer Nat 151:29-45

Tutin CEG (2000) Ecologie et organisation des primates de la foret tropicale africaine: aide a la comprehension de la transmission des retrovirus. Forets Tropicales et emergences virales 93:157-161

Walsh PD, Abernethy KA, Bermejo M, Beyersk R, De Wachter P, Akou ME, Huljbregis B, Mambounga DI, Toham AK, Kilbourn AM, Lahm SA, Latour S, Maisels F, Mbina C, Mihindou Y, Obiang SN, Effa EN, Starkey MP, Telfer P, Thibault M, Tutin CEG, White LJ T, Wilkie DS (2003) Catastrophic ape decline in western equatorial Africa. Nature 422:611-614

Walsh PD, Biek R, Real LA (2005) Wave-like spread of Ebola Zaire. PLoS Biol 3:e371

Walsh PD, Breuer T, Sanz C, Morgan D, Doran-Sheehy D (2007) Potential for Ebola transmission between gorilla and chimpanzee social groups. Am Nat 169:684-689

Watts DP (1989) Infanticide in mountain gorillas: new cases and a reconsideration of the evidence. Ethology $81: 1-18$

Watts DP (1991) Mountain gorilla reproduction and sexual behavior. Am J Primatol 24:211-226

Woolhouse MEJ, Haydon DT, Antia R (2005) Emerging pathogens: the epidemiology and evolution of species jumps. Trends Ecol Evol 20:238-244

Wrangham RW (2000) Why are male chimpanzees more gregarious than mothers? A scramble competition hypothesis. In: Kappeler PM (ed) Primate males. Cambridge University Press, Cambridge, pp 248-258

Yamagiwa J, Kahekwa J (2001) Dispersal patterns, group structure and reproductive parameters of eastern lowland gorillas at Kahuzi in the absence of infanticide. In: Robbins MM, Sicotte P, Stewart KJ (eds) Mountain gorillas: three decades of research at karisoke. Cambridge University Press, Cambridge, pp $89-122$ 